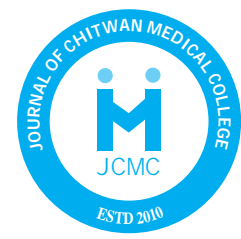

Journal of Chitwan Medical College 2017; 7(22): 62-65

Available online at: www.jcmc.cmc.edu.np

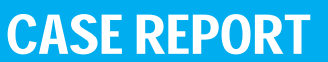

\title{
GOLDENHAR SYNDROME: A CASE REPORT
}

\author{
Sabin Sahu ${ }^{1}$, Kanchan Prakash Paudyal ${ }^{1}$, Bijendra Kumar Yadav ${ }^{2}$ \\ ${ }^{1}$ Sagarmatha Choudhary Eye Hospital, Lahan - 3, Siraha, Nepal. \\ ${ }^{2}$ Jinis Hospital, Lahan, Siraha, Nepal. \\ *Correspondence to: Dr Sabin Sahu, Sagarmatha Choudhary Eye Hospital, Lahan, Siraha, Nepal. \\ E mail: sabinsahu@gmail.com
}

\begin{abstract}
BACKGROUND: Traumatic dental injuries (TDI) are injuries involving the teeth and alveolar process of the jaw. TDIs are generally sudden and accidental and often require emergency care. Most TDIs represent dental emergencies that require immediate assessment and management. Immediate and proper management is required to minimize complications. There is a lack of studies related to TDIs in Nepal. The aim of this study was to investigate the treatment of TDIs and its associated factors in patients treated at, School of Dental Sciences, Chitwan Medical College (CMC), over a one-year period from January 2014 to December 2014. METHOD: All patients with TDIs, who attended the Department of Conservative Dentistry and Endodontics, School of Dental Sciences, CMC, over a one-year period from January 2014 to December 2014, were included in the study. RESULTS: A total of 60 patients ( 39 males [65\%], 21 females [35\%]) with a total no of 183 traumatized permanent teeth were analyzed in this study. With regard to the treatment of TDls, the most common treatment provided was root canal treatment (25\%), followed by composite resin restorations (24\%), splinting (10\%), direct pulp capping $(4.9 \%)$, repositioning and splinting (3.27\%). Follow-up and monitoring only was done in $(32 \%)$ of tooth without any direct treatment. CONCLUSION: Root canal treatment was the most common treatment provided although most of the injuries were uncomplicated crown fracture. Therefore effective and timely treatment of TDIs with follow up appointments is required to reduce or avoid complications. This study emphasizes the importance of timely treatment of TDIs.
\end{abstract}

Keywords: Dental Trauma, Tooth Injuries, Traumatic dental injuries, Treatment.

\section{INTRODUCTION}

Goldenhar syndrome is a rare congenital developmental anomaly involving the first and second brachial arches. It is also known as the oculo-auriculo-vertebral syndrome because of the association of eye anomalies with or without vertebral and ear abnormalities. It was first described in 1952 by ophthalmologist Maurice Goldenhar. ${ }^{1}$ The condition is characterized by multiple anomalies ranging from facial abnormalities, ear abnormalities, eye abnormalities and vertebral anomalies. In addition, cardiovascular, respiratory, central nervous system and genitourinary malformations have been associated with Goldenhar syndrome. The incidence of Goldenhar syndrome have been reported to range from 1:3500 to 1:5600 live births with a male to female ratio of 3:2. The exact etiology of the disease is not well understood. Most of the cases are seen as sporadic. However, autosomal dominant, autosomal recessive and multifactorial modes of inheritance have also been suggested. ${ }^{2}$

\section{CASE REPORT}

A 2-year-old male child, born of non-consanguineous marriage, was brought to ophthalmology clinic with complaint of mass in the left eye since birth. The child was full-term normal delivery with no perinatal complications and there was no history of maternal illness during pregnancy. On examination, the patient follows and fixates to light. Full ocular motility was evident in both eyes. Left eye had limbal dermoid at 6 o'clock position (Figure 1A) and epibulbar lipodermoid with hair on the surface on the supero-temporal aspect (Figure 1B). There was 
no evidence of any coloboma or microphthalmia. Rest of the ocular examinations in left eye and right eye were within normal limit. The patient also had subtle hemifacial microsomia, with the left side of the face being slightly smaller than the right, defective nasal alae in left side and pre-auricular skin tags bilaterally (Figure 2). The cycloplegic refraction revealed against the rule astigmatism of 4 dioptres in left eye and right eye had no refractive error.

Figure 1. Anterior segment photograph of left eye showing limbal demoid at inferior limbus (1A) and epibulbar lipodermoid with hair on the surface superotemporally (1B).
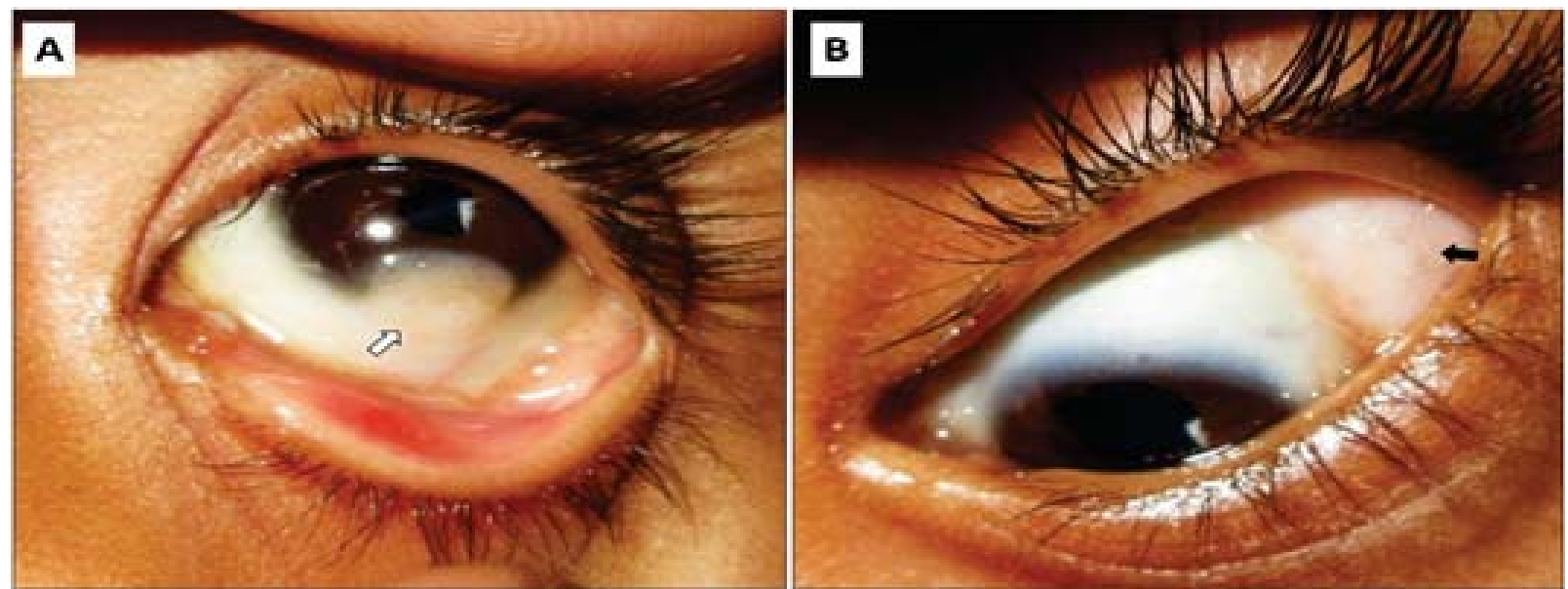

Figure 2. External photograph of patient showing left sided subtle hemifacial microsomia, defective nasal alae (2A) and pre-auricular skin tags (2B \& 2C).
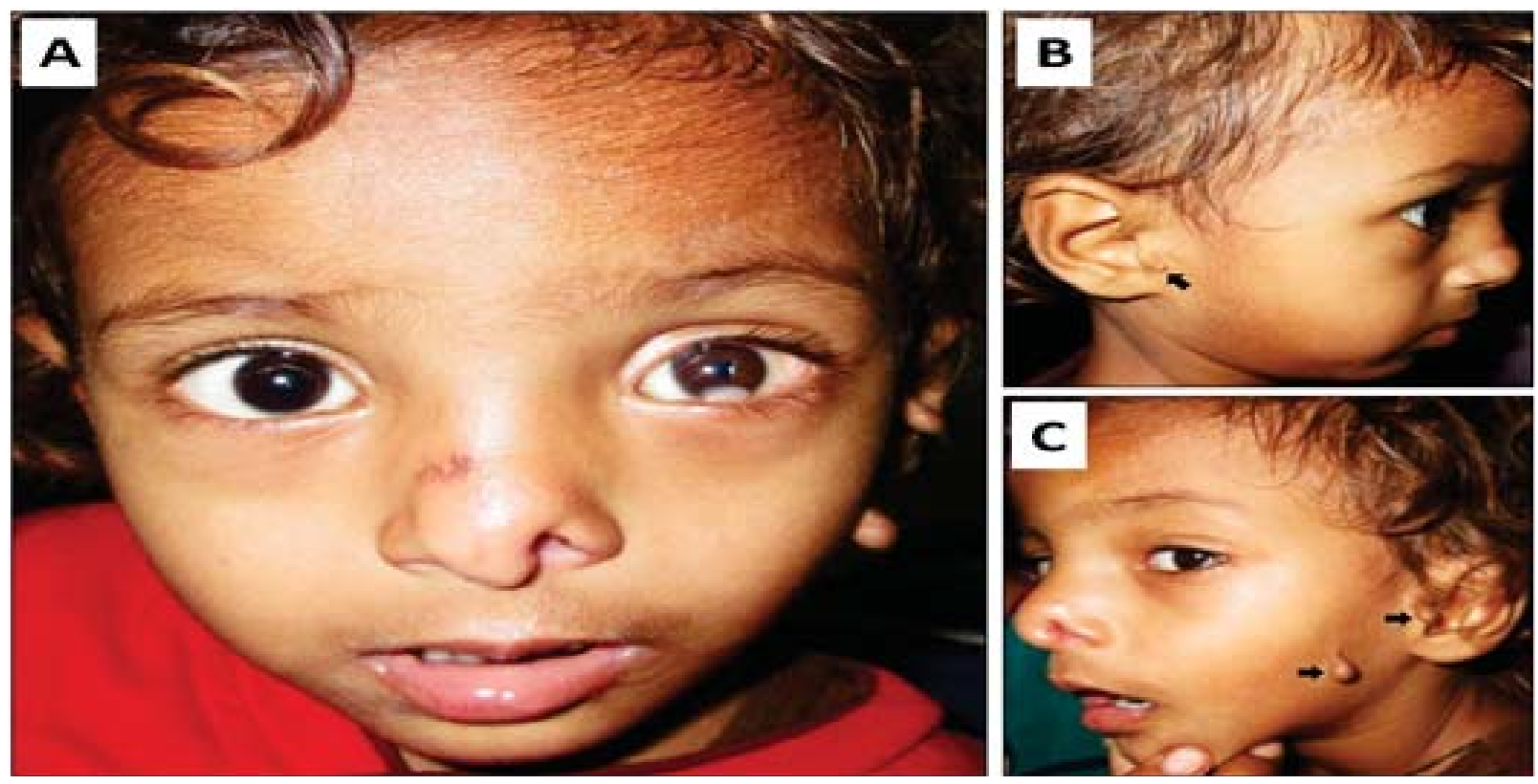

Systemic evaluation by pediatrician revealed right sided indirect inguinal hernia and no vertebral, cardiovascular, respiratory, genitourinary or central nervous system abnormalities were seen. The patient's mental and physical development was normal for his age. Electrocardiogram and chest X-ray and X-ray cervical spine were within normal limits for age. Ear examination didn't reveal any deformity or microtia. Hearing evaluations on two different occasions were normal. 
The limbal dermoid and the lipodermoid excision was done under general anesthesia. Preanesthetic assessment showed adequate mouth opening, Mallampatti class II, normal thyromental distance and neck movements. Available difficult airway cart was kept ready. The general anesthesia was performed under laryngeal mask airway with use of intravenous propofol for induction, fentanyl for analgesia and isoflurane for maintenance of anesthesia. The surgical procedure was smooth, uneventful and lasted for 20 minutes.

The patient recovered well and healed with minimal corneal scar (Figure 3). Postoperative astigmatism reduced to 2 dioptre against the rule astigmatism. The spectacle correction was provided and occlusion therapy started to prevent amblyopia of the left eye. We continue to follow up the patient periodically and there is no recurrence of limbal dermoid in almost one year of follow up.

Figure 3. Appearance one month after surgical excision of limbal dermoid and lipodermoid (3A) with minimal corneal scar (3B)
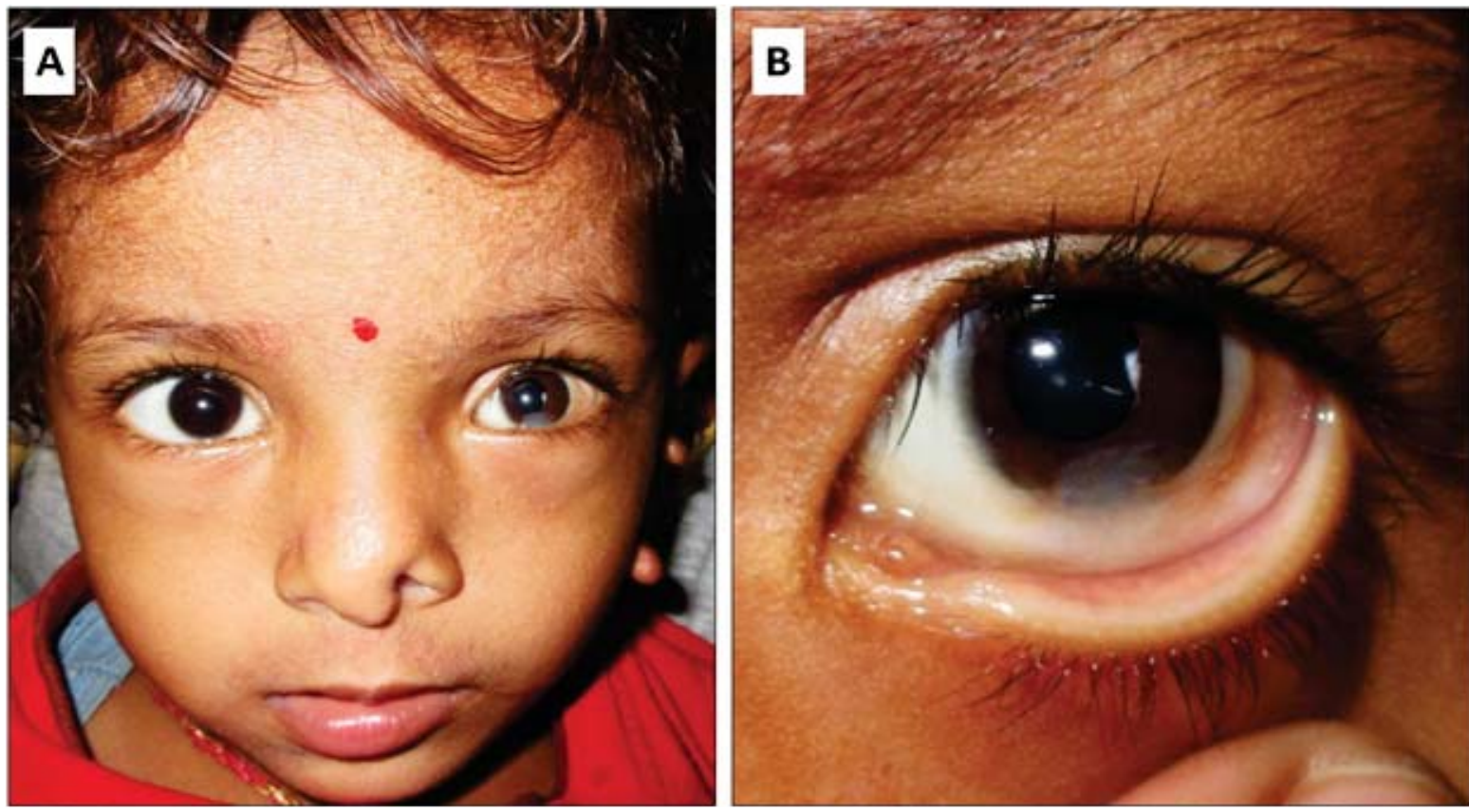

\section{DISCUSSION}

The spectrum of goldenhar syndrome abnormalities may range from mild to severe forms. Though the exact etiology is not known, the proposed pathophysiologic mechanisms include abnormal embryonic vascular supply, or disruption to the mesoderm, among other factors, leading to a defective formation of the brachial arches. 3 The abnormalities are mostly unilateral (85\% of cases) and the right side is more frequently affected. 4 The main ocular feature of goldenhar syndrome is the epibulbar choriostoma in form of a limbal dermoid or a lipodermoid, which is seen in 60\% cases. 5,6 Vertebral anomalies (scoliosis, wedge shaped vertebrae, fusion of several vertebrae, semi-vertebrae, spina bifida) are seen in $40-60 \%$ and ear anomalies (anotia, microtia, preauricular appendages, preacuricular fistulas, middle and inner ear abnormalities, dysmorphic ear with or without hearing loss) are seen in $40 \%$ of cases. 6,7 Other ocular features such as microphthalmia, anophthalmia, strabismus, epicanthal folds, eyelid coloboma, coloboma or iris or choroid, anomalies of lacrimal drainage system, retina and optic nerve anomalies, glaucoma have also been reported. 7,8,9 In our case, no ocular abnormalities other than unilateral limbal dermoid and lipodermoid was found.

Airway management in patients with Goldenhar syndrome is usually difficult due to associated unilateral maxillary and mandibular hypoplasia and craniovertebral anomalies which often result in limitation of 
neck movements. Using flexible fiberoptic intubation under local anesthesia with or without sedation is the technique of choice for management of the anticipated difficult airway in the patient undergoing an elective procedure. 10 But in our case we were able to perform the surgery under general anesthesia with use of laryngeal mask airway.

The limbal dermoid is a benign tumor but may lead to serious ophthalmic consequences like induced astigmatism, amblyopia and strabismus. The epibulbar lipodermoids and dermoids of large size may obstruct the visual axis, cause limitation of ocular motility or hinder eyelid closure leading to exposure keratitis and corneal ulceration. The dermoid with lashes may cause constant rubbing of eye leading to symptoms of irritation, watering, discharge, secondary infections and corneal epithelial defects and ulcerations. The early diagnosis and surgical excision of limbal dermoid lesions may prevent amblyopia and strabismus especially in pediatric age group. In our case, the induced astigmatism reduced significantly after surgical excision of the limbal dermoid and lipodermoid.

The management of Goldenhar syndrome require a multidisciplinary approach involving ophthalmologists, pediatrician, otorhinolaryngologists, radiologists, orthodontist, dermatologists, orthopedician. The treatment of the condition varies with age, systemic associations and the severity of the disease. The timely ophthalmological management can improve cosmesis and prevent the visual consequences. Since the syndrome can present with wide spectrum of symptoms and systemic associations, awareness of such rare case is essential for early diagnosis and proper management.

\section{REFERENCES}

1. Goldenhar M. Associations malformatives de l'oeil et l'oreille, en particulier le syndrome dermoide epibulbaire-appendices auriculairesfistula auris congenita et ses relations avec la dysostose mandibulofaciale. J Genet Hum. 1952;1:243-282.

2. Kulkarni V, Shah MD, Parikh A. Goldenhar syndrome: A case report. J Postgrad Med.1985;31:177-9

3. Hartsfield JK. Review of the etiologic heterogeneity of the oculo-auriculo-vertebral spectrum (Hemifacial Microsomia). Orthod Craniofac Res. 2007;10:121-8.

4. Kokavec R. Goldenhar syndrome with various clinical manifestations. Cleft Palate Craniofac J. 2006 Sep;43(5):628-634.

5. Murat O, Mesut G, Aytekin G, Guven O-Goldenhar syndrome associated with bilateralocular choristomas and cardiac abnormalies. Eur J Gen Med 2004;1: 28-30.
6. Smith, D. W.: Recognizable patterns of human malformations. In, "Major Problems in Clinical Paediatrics," W. B. Saunders Company, Philadelphia, 1982, pp. 168-169.

7. Sharma JK, Pippal SK, Raghuvanshi SK Goldenhar Gorlin's syndrome. case report. Indien J Otolaryngol Head Neck Surg 2006;58: 97-101.

8. S. Bhallil, I. Benatiya, O. El Abdouni, B. Mahjoubi, and T. Hicham. Goldenhar syndrome: ocular features. Bull. Soc. belge Ophtalmol. 2010; 316, 17-20

9. Rao VA, Kaliaperumal S, Subramanyan T, Rao KR, Bhargavan R. Goldenhar's sequence with associated juvenile glaucoma in Turner's syndrome. Indian J Ophthalmol 2005;53:267-8

10. Khan WA, Salim B, Khan AA, Chughtai S. Anaesthetic Management in a Child with Goldenhar Syndrome. J Coll Physicians Surg Pak. 2017;27:S6-S7. 